\title{
Early mammalian development: from basic research to the clinic
}

\author{
Guest Editors \\ Aneta Suwińska and Anna Ajduk \\ Department of Embryology, Faculty of Biology, University of Warsaze, Warsaw, Poland \\ (iD) https:/ / orcid.org/0000-0001-8366-2197 (AS) \\ (iD) https://orcid.org/0000-0002-7262-1370 (AA)
}

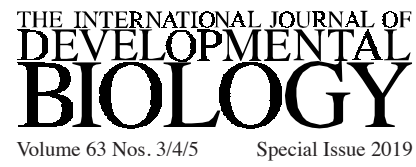





\section{Preface}

\section{Early mammalian development: from basic research to the clinic}

Preimplantation embryonic development lays the foundations for the future individual. Fertilization, cleavage, differentiation of the first embryonic cell lineages and implantation of the embryo into the maternal uterus are absolutely critical for proper embryogenesis.

Solving unanswered questions as well as creating new ideas and theories constitute the main axis of the basic research, which is driven by the curiosity of scientists and their desire to explore the unknown. We researchers have been exploring the development of mammalian embryos for decades, searching for the answer to the most fundamental question in the whole area of biology: how a complex organism derives from a single totipotent cell, a zygote. Due to obvious ethical concerns, animals, such as mice and, currently more and more often, cattle, pigs and rabbits, have become useful models for studying human embryonic development. Unprecedented advancement in cell and molecular biology techniques witnessed in the last years allows us to deepen our understanding of mammalian embryonic development. Specifically, the last decade has brought us a real explosion of new data identifying genes responsible for regulation of the preimplantation embryo development, obtained predominantly, but not exclusively, in the mouse. Extending our research to non-rodent animal models is nowadays crucial, as recent molecular studies have revealed unexpected differences between species and raised concerns regarding the extent to which we can confidently extrapolate data from mice to humans. We should bear it in mind because basic research often paves the way to practical applications, in vitro fertilization (IVF) being a perfect example here. The pioneering work of Pincus, Austin, Chang, McLaren, Tarkowski and many others on oocyte maturation, fertilization and embryo development, performed mostly on rabbit and mouse cells, led to the discoveries of Edwards, Steptoe and Purdy and, consequently, to the development of human ART (assisted reproductive technology).

Inviting experts to this Special Issue of The International fournal of Developmental Biology (Int. F. Dev. Biol.), we were hoping to provide an overview of the state-of-the-art knowledge on mammalian development, with a particular emphasis on application of the presented research in practice, especially in human ART. Over the years, IVF protocols have continued to evolve to improve their outcomes. However, despite this significant progress, there is still a lot to accomplish. For example, the current methods of assessing embryo viability provide a rough indication rather than a conclusive prediction of the developmental potential of individual embryos. Understanding the molecular mechanisms underlying cell cycle regulation and the first cell differentiation events in human preimplantation development is essential for optimization of the IVF procedure and establishment of more reliable protocols for embryo quality assessment. As tools introduced initially in animal models, such as genome editing, in vitro culture of post-implantation embryos and embryo-derived stem cells, have been recently applied to humans as well, this aim is closer than ever. New avenues for the direct exploration of human embryonic development have opened and all these novel technologies will hopefully help us to uncover causes of infertility and miscarriage. However, as always, new opportunities entail new challenges: the technological advancement enables developmental and reproductive scientists to look deeper and further than ever before, but at the same time pushes us towards inevitable ethical dilemmas. Nevertheless, it is difficult not to feel excited with the prospect of all the discoveries that the future may bring.

Aneta Suwinska and Anna Ajduk Warsaw, December 2018

Int. J. Dev. Biol. 63: 73-75 (2019)

https://doi.org/10.1387/ijdb.180409as 